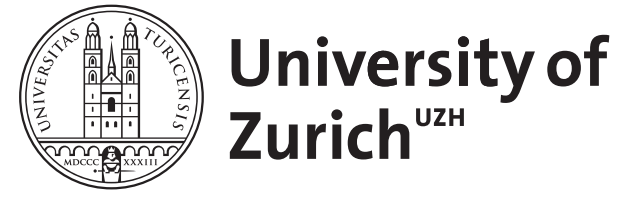

Main Library

Strickhofstrasse 39

CH-8057 Zurich

www.zora.uzh.ch

Year: 2017

\title{
Patient Blood Management: Der Standard heute
}

Kaserer, Alexander ; Stein, Philipp ; Spahn, Gabriela ; Spahn, Donat R

DOI: https://doi.org/10.1024/0040-5930/a000928

Posted at the Zurich Open Repository and Archive, University of Zurich

ZORA URL: https://doi.org/10.5167/uzh-145893

Journal Article

Accepted Version

Originally published at:

Kaserer, Alexander; Stein, Philipp; Spahn, Gabriela; Spahn, Donat R (2017). Patient Blood Management: Der Standard heute. Therapeutische Umschau. Revue thérapeutique, 74:369-376.

DOI: https://doi.org/10.1024/0040-5930/a000928 


\title{
Patient Blood Management: Der Standard heute
}

\author{
Alexander Kaserer, Philipp Stein, Gabriela H. Spahn, Donat R. Spahn \\ Institut für Anästhesiologie, Universität und Universitätsspital Zürich, Rämistrasse 100, \\ 8091 Zürich, Schweiz
}

\section{Korrespondenzadresse:}

Professor Dr. Donat R. Spahn, Direktor Institut für Anästhesiologie, Universität und Universitätsspital Zürich, Rämistrasse 100, 8091 Zürich, Schweiz. Email:

donat.spahn@usz.ch

\section{Zusammenfassung}

Das Patient Blood Management ist ein multidisziplinäres Behandlungskonzept, das vorrangig die Behandlung von Anämie und Eisenmangel, die Minimierung von Blutverlusten und die Erhöhung der Anämietoleranz beinhaltet. Die Anwendung des Patient Blood Management Konzeptes ist nicht nur für die perioperative Phase, sondern für alle Bereiche der Medizin indiziert, die sich mit der Behandlung mit Blut und Blutprodukten auseinandersetzen. Eine Vielzahl von Studien mit bis zu 605.000 Patienten haben die Sicherheit und Effektivität dieses Konzepts geprüft. Dabei konnten Fremdbluttransfusionen reduziert, finanzielle Ressourcen geschont und klinische, patientenbezogene Outcomeparameter, inklusive Morbidität und Mortalität, verbessert werden. Patient Blood Management ist daher zu einem Standard der Patientenversorgung geworden.

\section{Summary (English)}

Patient Blood Management is a multidisciplinary concept aiming at improving patient outcome by treatment of preoperative anemia and iron deficiency, minimizing perioperative blood loss and improving anemia tolerance. Patient Blood Management measures are also applicable in non-surgical domains. A multitude of studies with up to 605'000 patients have proven its safety and efficacy. Not only were transfusions reduced but also clinical and financial outcomes were significantly improved including reduced morbidity and mortality. Patient Blood Management thus has become a standard. 


\section{Einleitung}

Präoperativ weisen 20-40\% aller Patienten eine Anämie oder einen isolierten Eisenmangel auf.[1] Besonders Patienten mit einem Tumorleiden, okkulten Blutverlusten, einer chronisch entzündlichen Darmerkrankung, einer Niereninsuffizienz, einer Herzinsuffizienz oder eines komplexen entzündlichen Geschehens sind betroffen.[1]

Eine präoperative Anämie geht mit einer erhöhten Mortalität und vermehrten Komplikationen einher und zwar in der Allgemein- wie auch der Herz-Chirurgie.[2-4] Eine präoperative Anämie erhöht schliesslich das Risiko von Bluttransfusionen, was wiederum die Mortalität und die Komplikationsrate erhöht.[5] Die typischen Komplikationen von transfundierten Patienten sind Lungen- und Nieren-Schädigungen, vermehrte nosokomiale Infektionen, ein beschleunigtes Tumorwachstum und eine verlängerte Hospitalisation.[5]

Um diese Komplikationen zu vermeiden hat die Weltgesundheitsorganisation (WHO) im Jahre 2010 das Patient Blood Management Konzept angenommen und fordert seither alle 194 Mitgliedstaaten auf, dieses Konzept umzusetzen.[5] Das Patient Blood Management Konzept beruht auf 3 Säulen: Der präoperativen Detektion und Behandlung einer Anämie oder eines Eisenmangels, die Minimierung des perioperativen Blutverlusts und die Förderung der Anämie-Toleranz.[5, 6] Die Anämie ist in diesem Zusammenhang definiert durch ein Hämoglobin von < $130 \mathrm{~g} / \mathrm{L}$ und zwar für Männer und Frauen.

\section{Säule}

Prävalenz und Bedeutung der präoperativen Anämie oder eines Eisenmangels

Eine präoperative Anämie tritt in $20-40 \%$ der elektiv-operierten Patienten auf.[2] Ebenso häufig findet sich ein isolierter Eisenmangel.[1] Eine unbehandelte Anämie, selbst im geringen Ausmaß, geht im Rahmen einer bevorstehenden Operation mit einem erhöhten Risiko für Komplikationen und Sterblichkeit einher.[7] Unabhängig vom Einsatz von Fremdbluttransfusionen konnte mehrfach gezeigt werden, dass eine Anämie mit einem erhöhten Risiko für postoperative Morbidität und Mortalität assoziiert ist.[1, 2, 8, 9]

\section{Anämieformen}

Anämien lassen sich anhand ihrer Morphologie (MCV) in mikro-, makro- normozytäre Anämien, anhand ihres Hämoglobin-Gehaltes $(\mathrm{MCH})$ in normo-, hypo- hyperchrome Anämien und anhand ihrer Erythropoese in hypo-, normo- oder hyperregenerative Anämien einteilen. Daneben unterscheidet man weiter zwischen akuten und chronischen, sowie angeborenen und erworbenen Anämien. Im Folgenden werden die wichtigsten Anämieformen im perioperativen Setting kurz erläutert: 
Eisenmangelanämie: Die Eisenmangelanämie ist die häufigste Form der Anämie, deren Anteil je nach Land zwischen 3\%-65\% variiert.[10] In Industrieländern handelt es sich bei etwa einem Drittel der präoperativ diagnostizierten Anämien um eine Eisenmangelanämie.[2, 11] Laborchemisch hinweisgebend dafür sind ein verringertes Hämoglobin, $\mathrm{MCV}, \mathrm{MCH}$, Ferritin, sowie eine erniedrigte Transferrinsättigung. Die Eisenmangelanämie wird daher der mikrozytären, hypochromen Anämie zugeordnet. Unter effektiver Eisentherapie - aufgrund des Zeitdrucks bevorzugt intravenös - steigt die Hämoglobinkonzentration innerhalb weniger Tage signifikant an.[12, 13]

Renale Anämie: Ursache einer renalen Anämie ist eine verminderte Synthese von Erythropoetin. Hinweisgebend ist neben einer normochromen, normozytären Anämie eine erhöhte Serumkreatininkonzentration mit einer reduzierten Kreatininclearance. Häufig liegt gleichzeitig ein Eisenmangel vor. Die Therapie der renalen Anämie erfolgt mittels rekombinantem, humanem Erythropoetin in Kombination mit intravenösem Eisen.

Anämie der chronischen Erkrankung: Die Anämie der chronischen Erkrankung kann im Verlauf einer chronischen entzündlichen Erkrankung, bei Infektionskrankheiten und malignen Erkrankungen begleitend auftreten. Ursächlich dafür ist eine inadäquate Eisenfreisetzung aus häufig normal gefüllten Eisenspeichern. Laborchemisch findet man ein erniedrigtes Hämoglobin, ein normales $\mathrm{MCV}$ und $\mathrm{MCH}$, sowie eine erniedrigte Transferrinsättigung. Das Serumferritin als Akut-Phase-Protein ist häufig erhöht. Die Behandlung erfolgt durch die Verabreichung von rekombinantem humanen Erythropoetin meist in Kombination mit intravenösem Eisen.

\section{Präoperative Anämiebehandlung}

Für eine optimale Vorbereitung stellen sich Patienten 3-4 Wochen vor dem geplanten, elektiven Eingriff in der Anästhesie-Sprechstunde vor. Ziel dabei ist es, eine zu diesem Zeitpunkt vorhandene Anämie zu erkennen und weiter zu differenzieren (Eisenmangel, renale Anämie etc.). Dazu erfolgt laborchemisch die Bestimmung der Hb-Konzentration, MCV, MCH, Transferrinsättigung, Serumferritinkonzentration und des Serumkreatinins. Die Behandlung erfolgt gemäss den vorgängig beschriebenen Prinzipen. Eine präoperative Fremdblut-Transfusion ist keine Option.[5] 
Die präoperative Anämie-Behandlung im Rahmen des Patient Blood Management Konzepts bewirkt eine

- Reduktion der Fremdbluttransfusionen [11, 14-16]

- Verkürzung des Krankenhausaufenthaltes [9, 14, 16]

- Reduktion nosokomial erworbener Infektionen [15]

- Reduktion von Herzinfarkt oder Schlaganfall [15]

- Verminderung der Mortalität $[2,9,15,17]$

Am Institut für Anästhesiologie des Universitätsspitals Zürich werden Patienten, bei denen eine Operation mit hohem Blutungsrisiko (Transfusions-Risiko $>10 \%$ oder erwarteter Blutverlust > $500 \mathrm{ml}$ ) längerfristig geplant ist, gemäß dem untenstehenden Schema abgeklärt und behandelt: 
Abbildung 1: Schema zur Evaluation und Behandlung einer präoperativen Anämie des Instituts für Anästhesiologie am UniversitätsSpital Zürich.[17]

$\mathrm{Hb}=$ Hämoglobin; TSAT = Transferinsättigung; CRP = C-reaktives Protein; EPO $\alpha=$ Erythropoetin alpha;

\section{Evaluation und Behandlung der präoperativen Anämie}

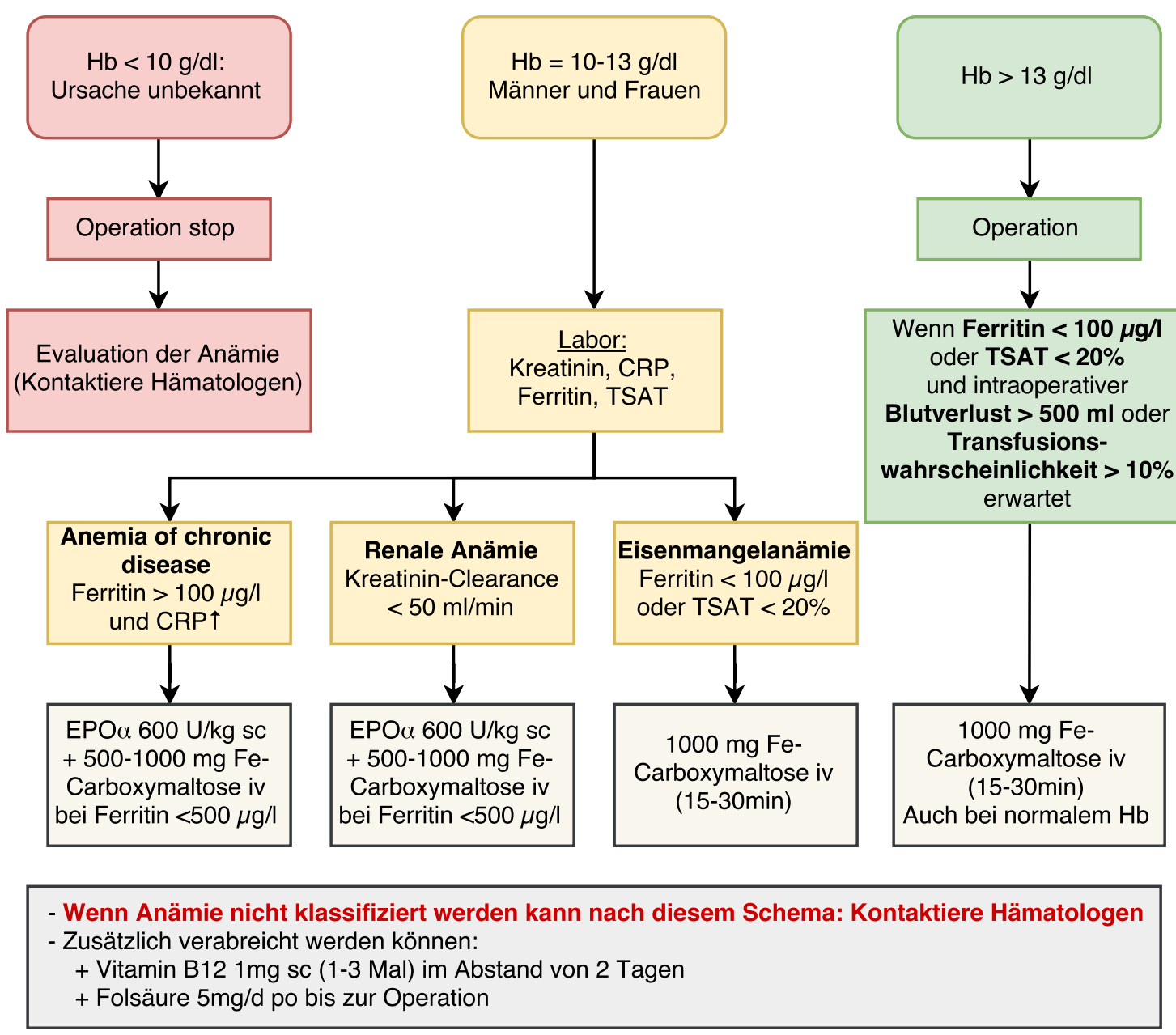




\section{Säule}

Die zweite Säule des Patient Blood Management beinhaltet sämtliche Massnahmen die während einer Operation oder während eines Eingriffs zur Vermeidung von Blutverlust führen. Veränderte chirurgische Techniken insbesondere bei der Blutstillung haben dazu geführt, dass grosse Operationen in der Herzchirurgie und Transplantationschirurgie auch ohne die Verwendung bzw. Transfusion von Fremdblut und Fremdblutprodukten möglich sind.

Bei Schwerverletzten hat ein Paradigmenwechsel bei anästhesiologischem und unfallchirurgischem Management dazu geführt, dass Transfusionen von Fremdblut, insbesondere Massivtransfusionen ( $>10$ Erythrozytenkonzentrate bis Aufnahme Intensivstation) und Transfusionen von Plasma massiv und statistisch signifikant reduziert werden konnten (Tabelle 1).[18]

Tabelle 1: Signifikante klinische Benefits bei Traumapatienten (alle $p \leq 0.001$ ) [18] $\mathrm{PBM}=$ Patient Blood Management, IPS = Intensiv-Pflege-Station

\begin{tabular}{|l|c|c|}
\hline Klinische Benefits durch PBM im Trauma Zentrum & vorher & nachher \\
\hline Reduktion der Massivtransfusionen bis Aufnahme IPS & $12 \%$ & $4 \%$ \\
\hline Reduktion der Transfusion von Erythrozytenkonzentraten bis Aufnahme IPS & $43 \%$ & $17 \%$ \\
\hline Reduktion der Transfusion von Frischplasma bis Aufnahme IPS & $31 \%$ & $6 \%$ \\
\hline $\begin{array}{l}\text { Reduktion der Mortalität nach 24h } \\
\text { und bis Spitalentlassung }\end{array}$ & $17 \%$ & $10 \%$ \\
\hline Reduktion der Tage auf der Intensivstation (Mittelwert) & $33 \%$ & $22 \%$ \\
\hline Reduktion der Tage mit kontrollierter Beatmung (Mittelwert) & 18.0 & 15.4 \\
\hline
\end{tabular}

\section{Cell salvage}

Intraoperativer Blutverlust oder traumatische Blutungen in Körperhöhlen können durch spezielle Absaugsysteme automatisch aufgearbeitet und die Erythrozyten dem Patienten re-transfundiert werden. Die Sicherheit und die Effizienz wurden in unzähligen Studien aus allen chirurgischen Spezialgebieten nachgewiesen.[19] Von entscheidender Bedeutung ist, dass das Blut aus einem nicht kontaminierten Gebiet gewonnen wird. Diese Verunreinigungen können sowohl mikrobiologisch sein (etwa durch traumatisch eröffnete Körperhöhlen, oder durch anderweitig infizierte Gebiete) oder durch Beimischung maligner Zellen bei onkologischer Chirurgie.

Der Einsatz von Geräten zur maschinellen Aufarbeitung von Blutverlust während einer Operation oder nach Trauma muss früh in das Behandlungskonzept integriert werden. Bei elektiven Operationen mit antizipiertem höheren Blutverlust muss diese Methode bereits vorab geplant werden. Bei akutem unerwartetem Blutverlust oder bei Traumapatienten 
muss die Methode zu einem frühen Zeitpunkt in Betracht gezogen werden, etwa vor einer notfallmässigen Laparotomie oder Thorakotomie, da diese initialen hohen Verluste an eigenem rotem Zellvolumen sonst verloren gehen.

\section{Gerinnungsmanagement}

Ein funktionierendes Gerinnungssystem ist grundlegende Voraussetzung, um den perioperativen Blutverlust zu minimieren. Klassischerweise wird die primäre Hämostase der zellulären Antwort zugeschrieben, also der Interaktion von Blutplättchen mit dem Endothel und dem Gewebe. Die sekundäre bzw. plasmatische Gerinnung wird klassischerweise in einen extrinsischen und in einen intrinsischen Weg unterteilt. Am intrinsischen Weg sind die Gerinnungsfaktoren XII, XI, IX und VIII beteiligt, der extrinsische Weg wird gestartet durch Aktivierung von Faktor VII durch den „tissue factor“. Beide Wege aktivieren Faktor X, der stark vereinfacht beschrieben Prothrombin (Faktor II) aktiviert. Extrinsischer und intrinsischer Weg laufen im Körper keinesfalls getrennt, sondern parallel ab. Die klassische Unterscheidung beruht auf den verfügbaren Labortests, welche diese Wege in vitro separat aktivieren. Eine Verlängerung dieser Gerinnungstests, Prothombinzeit (PT) für den extrinsischen Weg und partielle Thromboplastinzeit (PTT) für den intrinsischen Weg, korrelieren deshalb schlecht mit dem Phänotyp einer klinischen Blutung des Patienten.

Eine ungezielte Transfusion von allogenen Blutprodukten wie Frischplasma (FFP), Thrombozytenkonzentraten und Erythrozytenkonzentraten in einem festen Verhältnis wird zwar in gewissen Teilen dieser Welt nach wie vor praktiziert, eine solche ist aber heute nicht mehr „state-of-the-art".

Ein moderner Gerinnungs-Algorithmus beruht heute auf der Physiologie der Gerinnung und beinhaltet patientennahe (,point-of-care“) Gerinnungsdiagnostik und die gezielte Verabreichung von Gerinnungsfaktoren gemäss einem klinik-internen Algorithmus. Aufgrund eines solchen Gerinnungs-Algorithmus [20] ist es möglich, selbst bei schwerverletzten und blutenden Patienten weitgehend auf die Transfusion von allogenem Fremdplasma zu verzichten.[18]

Nach Erhebung einer detaillierten Gerinnungs- und Medikamentenanamnese werden klassische Gerinnungstests (PT, PTT) und „point of care“ Diagnostik zur perioperativen Monitorisierung der Gerinnung eingesetzt. Diese „point of care“ Diagnostik umfasst viskoelastischen Gerinnungstests wie Thromboelastometrie (ROTEM ${ }^{\circledR}$, TEM International $\mathrm{GmbH}$, München, Deutschland) und eine Thrombozytenfunktions_Analyse (z.B. Multiplate ®, Roche Diagnostics International Ltd, Rotkreuz, Schweiz).

Die Thromboelastometrie ermöglicht das Detektieren von (Hyper)fibrinolyse, niedrigen Fibrinogenspiegeln, einer verzögerten Gerinnungsinitiierung und niedrigen Thrombozytenzahlen. Hierfür werden standardmässig die Kanäle EXTEM (aktiviert durch 
„tissue factor“), INTEM (Oberflächenaktivierung), FIBTEM (aktiviert durch „tissue factor“ und Hemmung der Thrombozyten durch Cytochalasin D) und APTEM (Zugabe von Aprotinin zur Fibrinolysehemmung) bestimmt. Der FIBTEM Wert („maximum clot firmness“) als Surrogat für den funktionellen Fibrinogenspiegel erlaubt die frühe Substitution von Fibrinogen Konzentrat, da Fibrinogen bei einer aktiven Blutung als erstes kritisch tiefe Werte erreicht.[21] Eine Substitution von Fibrinogen (2-4 Gramm beim Erwachsenen) sollte bei einem FIBTEM $\leq 7 \mathrm{~mm}$ erfolgen. Die frühzeitige Gabe des Fibrinolysehemmers Tranexamsäure (TXA) hat bei Traumapatienten zu einer Reduktion der Mortalität geführt.[22] Ebenso führt die Gabe von TXA bei postpartaler Blutung zu einer Reduktion der maternalen Sterblichkeit ohne thromboembolische Nebenwirkungen.[23] Bei signifikanter Blutung wird deshalb 1 Gramm TXA „blind“ gegeben, dies wird u.a. in den „European Trauma Treatment Guidelines“ (ETTG) entsprechend empfohlen.[24] Der Gerinnungsalgorithmus des Instituts für Anästhesiologie des Universitätsspitals Zürich beschreibt das perioperative oder periinterventionelle Gerinnungsmanagement von Patienten. Ziele sind Erhalt bzw. Wiederherstellung einer normalen Metabolik, permissive Hypotension bis zur chirurgischen Blutungskontrolle, (empirische) Behandlung einer Fibrinolyse, sowie Bestimmung und Substitution von Gerinnungsfaktoren. Allogene Blutund Plasmaprodukte werden sehr restriktiv und nur nach vorheriger Messung zielgerichtet eingesetzt. Volumenmanagement erfolgt mit balancierten kristalloiden Infusionslösungen. In Ausnahmefällen, bei speziellen Indikationen und nach Rücksprache mit Experten der Hämatologie, können von Willebrand Konzentrat oder in seltenen Fällen rekombinanter, aktivierter Faktor VII erwogen werden. Der Gerinnungsalgorithmus ist in Tabelle 2 zusammengefasst. Ein sehr ähnlicher Algorithmus zur Transfusion von Blutprodukten und zur Gerinnungstherapie bei massiver Blutung wurde kürzlich von der Schweizerischen Gesellschaft für Anästhesie und Reanimation (SGAR) als Empfehlung veröffentlicht.[25] 
Tabelle 2: Gerinnungsalgorithmus des Instituts für Anästhesiologie, UniversitätsSpital Zürich 2017

\begin{tabular}{|c|c|}
\hline Anamnese & "Point of care" und Gerinnungslabor \\
\hline $\begin{array}{l}\text { 1. Medikation } \\
\text { - Thrombozytenaggregationshemmung } \\
\text { - Heparin } \\
\text { - OAK (Vitamin-K Antagonisten, Xa oder Ila } \\
\text { Hemmer) } \\
\text { 2. Vorbestehende Laborwerte } \\
\text { 3. Vorerkrankungen (z.B.): } \\
\text { - HIT } \\
\text { - Willebrand-Jürgens-Syndrom } \\
\text { - Lebererkrankungen }\end{array}$ & $\begin{array}{l}\text { 1. ROTEM® (EXTEM, INTEM, FIBTEM, } \\
\text { APTEM), HEPTEM bei Verwendung von } \\
\text { Heparin } \\
\text { 2. Gerinnungslabor } \\
\text { • Anti Xa (Screening nach Heparin und } \\
\quad \text { Xa Hemmern) } \\
\text { - TZ (Screening nach Dabigatran) } \\
\text { - INR (Screening nach Vitamin-K } \\
\quad \text { Antagonisten oder Faktorenmangel), } \\
\text { CoaguChek® } \\
\text { - Faktor V (Leberversagen oder } \\
\quad \text { Faktorenmangel) } \\
\text { - Faktor XIII (Faktorenmangel) } \\
\text { 3. Impedanz Aggregometrie bei } \\
\text { Thrombozytenaggregationshemmung }\end{array}$ \\
\hline \multicolumn{2}{|l|}{ Physiologie (Zielparameter) } \\
\hline $\begin{array}{ll}\text { - } & \text { Normothermie }\left(\geq 35.0^{\circ} \mathrm{C}\right) \\
\text { - } & \text { Normokalzämie }\left(\mathrm{Ca}^{2+} \geq 1.15 \mathrm{mmol} / \mathrm{l}\right) \\
\text { - } & \text { Normaler Säurebasestatus }(\mathrm{pH}>7.2) \\
\text { - } & \text { Hämatokrit }(0.21-0.24) \\
\text { - } & \text { Permissive Hypotension } \\
\text { - MAP } 55-65 \mathrm{mmHg} \text { vor chirurgischer / } & \text { interventioneller Blutungskontrolle } \\
& \text { MAP } 80-90 \mathrm{mmHg} \text { bei } \\
& \text { Schädelhirntrauma }\end{array}$ & $\begin{array}{l}\text { Aktives Erwärmen } \\
\text { Calcium i.v. } \\
\text { Volumenmanagement mit balancierten } \\
\text { kristalloiden Lösungen. Gelatine kann } \\
\text { erwogen werden } \\
\text { EC Transfusion } \\
\text { Permissive Hypovolämie / Hypotension } \\
\text { Vasopressoren und Korrektur der Volämie }\end{array}$ \\
\hline \multicolumn{2}{|l|}{ Fibrinogenspiegel bestimmen } \\
\hline FIBTEM $\leq 7 \mathrm{~mm}$ & $\begin{array}{l}\text { Fibrinogen } 2 \text { - } 4 \text { g i.v. (nach } 6 \text { g Fibrinogen, } \\
\text { Gabe von Faktor XIII, } 15 \text { U/kg i.v.) }\end{array}$ \\
\hline \multicolumn{2}{|l|}{ Fibrinolyse detektieren } \\
\hline $\begin{array}{l}\text { EXTEM / INTEM: Lyse nach MCF und APTEM: } \\
\text { normal = Hyperfibrinolyse }\end{array}$ & $\begin{array}{l}\text { Tranexamsäure } \\
\text { - } \quad \text { Bolus: } 15 \mathrm{mg} / \mathrm{kg} \text { i.v. (empirische Gabe } \\
\text { evaluieren) } \\
\text { - } \quad \text { Dauerinfusion evaluieren } 1-2 \mathrm{mg} / \mathrm{kg} / \mathrm{h}\end{array}$ \\
\hline \multicolumn{2}{|l|}{ Fortbestehende Blutung } \\
\hline 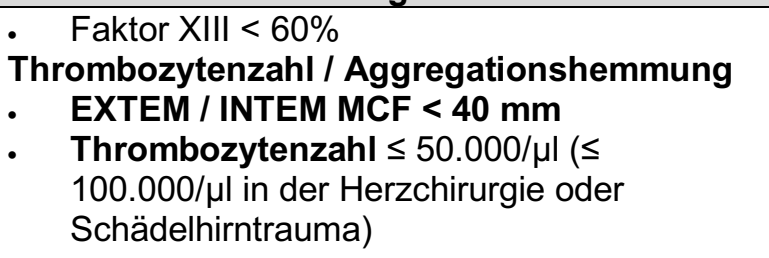 & $\begin{array}{l}\text { Faktor XIII, } 15 \mathrm{U} / \mathrm{kg} \text { i.v. } \\
\text { Thrombozytenkonzentrat }\end{array}$ \\
\hline $\begin{array}{l}\text { Thrombozytenfunktion } \\
\text { (Impedanz Aggregometie) }\end{array}$ & $\begin{array}{l}\text { Evaluation von Desmopressin } 0.3 \mu \mathrm{g} / \mathrm{kg} \\
(\max .16 \mu \mathrm{g}) \text { bei Aspirinwirkung }\end{array}$ \\
\hline $\begin{array}{ll}\text { - } & \text { INR }>2.3(\text { Quick < 30\%) } \\
\text { - } & \text { Faktor } \mathrm{V}<20 \%\end{array}$ & $\begin{array}{l}\text { 4-Faktor Prothrombin Komplex Konzentrat } \\
\text { (langsame Dauerinfusion von kleinen und } \\
\text { wiederholten Dosen - e.g. } 500 \mathrm{IU}) \\
\text { FFP (2-4 Beutel, } 15 \mathrm{ml} / \mathrm{kg})\end{array}$ \\
\hline Heparin detektieren & Antagonisiere Heparin \\
\hline $\begin{array}{l}\text { INTEM (CT/CFT) oder ACT verlängert und } \\
\text { HEPTEM oder Heparinase-ACT normal }\end{array}$ & Protamin $(1: 1)$ \\
\hline Spezialfälle & Evaluiere \\
\hline Expertenrat (Hämatologie) & $\begin{array}{l}\text { rFVIla } \\
\text { von Willebrand Faktor Konzentrat } \\
\text { Idarucizumab }\end{array}$ \\
\hline
\end{tabular}




\section{Antikoagulanzien}

Immer mehr Patienten haben durch steigendes Alter und die begleitende Morbidität eine Indikation für eine Antikoagulation etwa durch Vorhofflimmern, stattgehabte Thrombosen oder Lungenembolien.

Diese Antikoagulation kann einerseits durch Einnahme von Vitamin K Antagonisten erfolgen, andererseits zunehmend durch oral eingenommene direkte Antikoagulanzien (DOAK). Vitamin $\mathrm{K}$ Antagonisten führen zu einer verminderten Produktion der Gerinnungsfaktoren II, VII, IX, X sowie Protein S und C. DOAKs entfalten ihre Wirkung durch direkte Hemmung der Gerinnungsfaktors Xa (Rivaroxaban, Apixaban, Edoxaban) beziehungsweise des Gerinnungsfaktors Ila (Dabigatran).

Patienten mit gerinnungshemmender Medikation können bei Gewebeschaden im Rahmen einer Operation oder eines Traumas einen signifikant höheren Blutverlust aufweisen.

Bei elektiven oder semielektiven Eingriffen muss daher eine exakte vorherige Planung unter Einbeziehung aller involvierten und hierfür spezialisierten Disziplinen (Chirurgen, Anästhesisten) erfolgen. $\mathrm{Da}$ ein Absetzen beziehungsweise Überbrücken einer Antikoagulation ebenfalls relevante Risiken birgt, muss evaluiert werden, ob ein Eingriff mit geringen Blutungsrisiko auch unter Fortführen der Antikoagulation möglich ist. Falls dies aus chirurgischer Sicht nicht möglich ist, muss in enger Absprache mit Anästhesisten und Hausärzten (ggf. Hämatologen) das präoperative Procedere beziehungsweise Antikoagulation festgelegt werden. Die Schweizerische Gesellschaft für Anästhesiologie und Reanimation (SGAR) hat hierfür detaillierte Guidelines erarbeitet und aktualisiert diese regelmässig.

Grundsätzlich kann die Wirkung von Vitamin K Antagonisten durch deren Absetzen und/oder die Gabe von Vitamin K aufgehoben werden, dies in der Regel ohne Überbrückung durch therapeutische Gabe von niedermolekularen Heparinen. In einer Meta-Analyse wurde gezeigt, dass durch Überbrückung mit Heparinen keine thromboembolischen Ereignisse verhindert werden, aber die Inzidenz der Blutung signifikant erhöht wird.[26, 27] Auch die American Heart Association empfielt dieses Vorgehen.[28] Bei Patienten, die einem sehr hohen thromboembolischen Risiko unterliegen (spezielle mechanische Herzklappen, Lungenembolie innerhalb der letzten 6 Monate), kann ein Bridging mit Heparin nach Rücksprache mit der Anästhesie beziehungsweise Hämatologie erwogen werden. Dies erfolgt in der Regel durch therapeutische Gabe von niedermolekularen Heparinen. Hier ist insbesondere eine mögliche Akkumulation bei fortgeschrittener Niereninsuffizienz (errechnete glomeruläre Filtrationsrate $<30 \mathrm{ml} / \mathrm{min}$.) zu bedenken. 
Bei Patienten, die mit einem DOAK behandelt werden, wird dieser präoperativ gezielt abgesetzt und die Zeit bis zur Operation ebenfalls nicht mit Heparin überbrückt. Die SGAR hat hierzu detaillierte und aktualisierte Guidelines erarbeitet.

In einem Notfall, oder bei Patienten mit veränderter Kinetik (Alter > 80 Jahre, Leber-, Niereninsuffizient und Körpergewicht $<60 \mathrm{~kg}$ ) können konventionelle Gerinnungsparameter, oder Spiegelmessungen aller DOAKs direkte Hinweise auf die aktuelle antikoagulatorische Wirkung liefern. Der Quick Wert oder der „international normalized ratio“ (INR) liefert eine Aussage zur Wirkung von Vitamin K Antagonisten. Zur Antagonisierung der Wirkung wird bei Traumapatienten gemäss den European Trauma Treatment Guidelines die Gabe von Prothrombinkomplexkonzentraten empfohlen.[24] Eine solitär verlängerte Thrombinzeit kann einen Hinweis auf wirksame Spiegel von Dabigatran liefern, die dann durch eine Bestimmung der Hemmung von Faktor Ila qualifiziert und quantifiziert werden kann. Eine Antagonisierung von Dabigatran ist durch den spezifischen Antagonisten Idarucizumab möglich.[29] Dies bleibt speziellen Notfallindikationen nur nach Zuziehen von Experten (Hämatologie, Anästhesiologie) vorbehalten.

Nachweis einer anti-Faktor Xa Aktivität detektiert die direkten Faktor Xa Hemmer. Ein spezifischer Antagonist (Andexanet alpha) ist Gegenstand klinischer Studien.[30] Da bisher jedoch keine Zulassung der Arzneimittelbehörden vorliegt, ist aktuell noch keine spezifische Antagonisierung der Faktor Xa Hemmer möglich. Empfohlen wird die Behandlung mit Tranexamsäure und 4-Faktor Prothrombinkomplex Konzentrat.[24]

Für weitere, detaillierte Informationen bezüglich der Strategien zum Umgang mit antithrombotischen Medikamenten im perioperativen Umfeld möchten wir auf den Artikel von Timur Yurttas und Miodrag Filipovic verweisen.

\section{Säule}

\section{Akute Anämie}

Grundlegender Zweck des Sauerstofftransports im Körper ist es, den Geweben einen oxydativen Stoffwechsel zu ermöglichen. Das Sauerstoffangebot des Körpers ist das mathematische Produkt von Herzzeitvolumen und Sauerstoffgehalt des Blutes. Der Hämoglobinwert ist eine Konzentration $(\mathrm{g} / \mathrm{L})$ und proportional $\mathrm{zu}$ der insgesamt an Hämoglobin gebundenen Sauerstoffmenge. Solange physiologisch (durch ausreichende Vorlast) ein suffizientes Herzzeitvolumen vorhanden ist, kann durch dessen Steigerung auch ein niedriger Sauerstoffgehalt des Blutes (bei Anämie) kompensiert werden. Dies geschieht primär durch Steigerung der Inotropie am Herzen, sekundär durch eine Erhöhung der Herzfrequenz.[31] Durch diese Kompensationsmechanismen können auch sehr tiefe akute Hämoglobinwerte überlebt werden.[32] 


\section{Transfusions-Trigger}

Restriktive Hb-Transfusions-Trigger sind heute Standard.[33-35] Das Konzept der Patient Blood Managements zielt allerdings darauf ab, das $\mathrm{Hb}$ präoperativ zu normalisieren, den perioperativen Blutverlust zu minimieren, dass der Patient gar nie in eine Situation kommt, in welcher eine Transfusion diskutiert werden müsste.

\section{Erfolg des Patient Blood Managements}

Der Erfolg des Patient Blood Managements ist vielfach nachgewiesen mit Studien mit bis zu 605'000 Patienten.[15, 19, 35] In allen grossen Studien konnten nach Einführung des Patient Blood Managements nicht nur die Transfusionen massiv gesenkt werden, sondern auch die Komplikationsrate, die Hospitalistations-Dauer und die Mortalität (Abbildung 2). Ebenso konnten die Behandlungskosten deutlich gesenkt werden.[15, 35]

Abbildung 2: Schlüsselergebnisse der Implementierung des Patient Blood Managements.[15]

\section{Schlüsselergebnisse des Patient Blood Managements}

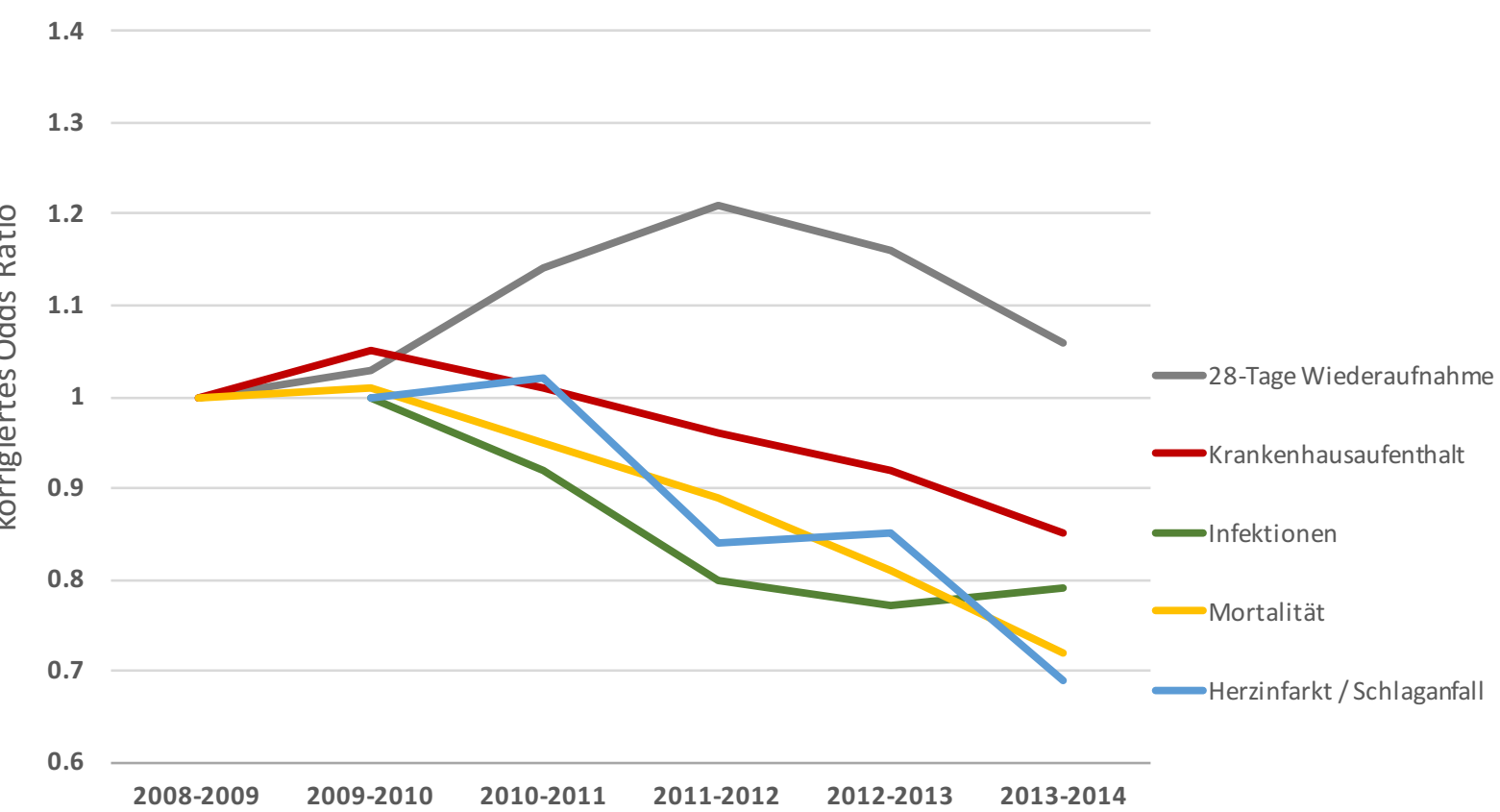




\section{Referenzen}

1. Munoz, M., Acheson, A.G., Auerbach, M., Besser, M., Habler, O., Kehlet, H., et al., International consensus statement on the peri-operative management of anaemia and iron deficiency. Anaesthesia, 2017. 72(2): p. 233-247.

2. Musallam, K.M., Tamim, H.M., Richards, T., Spahn, D.R., Rosendaal, F.R., Habbal, A., et al., Preoperative anaemia and postoperative outcomes in non-cardiac surgery: a retrospective cohort study. Lancet, 2011. 378(9800): p. 1396-407.

3. Klein, A.A., Collier, T.J., Brar, M.S., Evans, C., Hallward, G., Fletcher, S.N., et al., The incidence and importance of anaemia in patients undergoing cardiac surgery in the UK - the first Association of Cardiothoracic Anaesthetists national audit. Anaesthesia, 2016. 71(6): p. 627-35.

4. Baron, D.M., Hochrieser, H., Posch, M., Metnitz, B., Rhodes, A., Moreno, R.P., et al., Preoperative anaemia is associated with poor clinical outcome in non-cardiac surgery patients. Br J Anaesth, 2014. 113(3): p. 416-23.

5. Spahn, D.R., Goodnough, L.T., Alternatives to blood transfusion. Lancet, 2013. 381(9880): p. 1855-65.

6. Williamson, L.M., Devine, D.V., Challenges in the management of the blood supply. Lancet, 2013. 381(9880): p. 1866-75.

7. Gombotz, H., Rehak, P.H., Shander, A., Hofmann, A., The second Austrian benchmark study for blood use in elective surgery: results and practice change. Transfusion, 2014. 54(10 Pt 2): p. 2646-57.

8. Baron, D.M., Hochrieser, H., Posch, M., Metnitz, B., Rhodes, A., Moreno, R.P., et al., Preoperative anaemia is associated with poor clinical outcome in non-cardiac surgery patients. Br J Anaesth, 2014. 113(3): p. 416-23. 
9. Gruson, K.I., Aharonoff, G.B., Egol, K.A., Zuckerman, J.D., Koval, K.J., The relationship between admission hemoglobin level and outcome after hip fracture. J Orthop Trauma, 2002. 16(1): p. 39-44.

10. Kassebaum, N.J., Jasrasaria, R., Naghavi, M., Wulf, S.K., Johns, N., Lozano, R., et al., A systematic analysis of global anemia burden from 1990 to 2010. Blood, 2014. 123(5): p. 615-24.

11. Piednoir, P., Allou, N., Driss, F., Longrois, D., Philip, I., Beaumont, C., et al., Preoperative iron deficiency increases transfusion requirements and fatigue in cardiac surgery patients: a prospective observational study. European journal of anaesthesiology, 2011. 28(11): p. 796-801.

12. Keating, G.M., Ferric carboxymaltose: a review of its use in iron deficiency. Drugs, 2015. 75(1): p. 101-27.

13. Van Wyck, D.B., Mangione, A., Morrison, J., Hadley, P.E., Jehle, J.A., Goodnough, L.T., Large-dose intravenous ferric carboxymaltose injection for iron deficiency anemia in heavy uterine bleeding: a randomized, controlled trial. Transfusion, 2009. 49(12): p. 2719-28.

14. Calleja, J.L., Delgado, S., del Val, A., Hervas, A., Larraona, J.L., Teran, A., et al., Ferric carboxymaltose reduces transfusions and hospital stay in patients with colon cancer and anemia. Int J Colorectal Dis, 2016. 31(3): p. 543-51.

15. Leahy, M.F., Hofmann, A., Towler, S., Trentino, K.M., Burrows, S.A., Swain, S.G., et al., Improved outcomes and reduced costs associated with a health-system-wide patient blood management program: a retrospective observational study in four major adult tertiary-care hospitals. Transfusion, 2017. 57(6): p. 1347-1358. 
16. Froessler, B., Palm, P., Weber, I., Hodyl, N.A., Singh, R., Murphy, E.M., The Important Role for Intravenous Iron in Perioperative Patient Blood Management in Major Abdominal Surgery: A Randomized Controlled Trial. Ann Surg, 2016. 264(1): p. 41-6.

17. Cappellini, M.D., Comin-Colet, J., de Francisco, A., Dignass, A., Doehner, W., C, S.P.L., et al., Iron deficiency across chronic inflammatory conditions: International expert opinion on definition, diagnosis, and management. American journal of hematology, 2017. 92(10): p. 1068-1078.

18. Stein, P., Kaserer, A., Sprengel, K., Wanner, G.A., Seifert, B., Theusinger, O.M., et al., Change of transfusion and treatment paradigm in major trauma patients. Anaesthesia, 2017. 72(11): p. 1317-1326.

19. Meybohm, P., Herrmann, E., Steinbicker, A.U., Wittmann, M., Gruenewald, M., Fischer, D., et al., Patient Blood Management is Associated With a Substantial Reduction of Red Blood Cell Utilization and Safe for Patient's Outcome: A Prospective, Multicenter Cohort Study With a Noninferiority Design. Ann Surg, 2016. 264(2): p. 203-11.

20. Stein, P., Kaserer, A., Spahn, G.H., Spahn, D.R., Point-of-Care Coagulation Monitoring in Trauma Patients. Semin Thromb Hemost, 2017. 43(4): p. 367-374.

21. Martini, W.Z., Chinkes, D.L., Sondeen, J., Dubick, M.A., Effects of hemorrhage and lactated Ringer's resuscitation on coagulation and fibrinogen metabolism in swine. Shock, 2006. 26(4): p. 396-401.

22. Roberts, I., Shakur, H., Coats, T., Hunt, B., Balogun, E., Barnetson, L., et al., The CRASH-2 trial: a randomised controlled trial and economic evaluation of the effects of tranexamic acid on death, vascular occlusive events and transfusion requirement in bleeding trauma patients. Health Technol Assess, 2013. 17(10): p. 1-79. 
23. Collaborators, W.T., Effect of early tranexamic acid administration on mortality, hysterectomy, and other morbidities in women with post-partum haemorrhage (WOMAN): an international, randomised, double-blind, placebo-controlled trial. Lancet, 2017. 389(10084): p. 2105-2116.

24. Rossaint, R., Bouillon, B., Cerny, V., Coats, T.J., Duranteau, J., Fernandez-Mondejar, E., et al., The European guideline on management of major bleeding and coagulopathy following trauma: fourth edition. Critical care, 2016. 20(1): p. 100.

25. Brüesch, M. SGAR Gerinnungs-Algorithmus. 2017; Available from: http://www.sgarssar.ch/fileadmin/user upload/Dokumente/AG Gerinnungs-

Blutproduktemanagement/Standard Algorithmus 22032017.pdf.

26. Albaladejo, P., Samama, C.M., Sie, P., Kauffmann, S., Memier, V., Suchon, P., et al., Management of Severe Bleeding in Patients Treated with Direct Oral Anticoagulants: An Observational Registry Analysis. Anesthesiology, 2017. 127(1): p. $111-120$.

27. Hovaguimian, F., Koppel, S., Spahn, D.R., Safety of Anticoagulation Interruption in Patients Undergoing Surgery or Invasive Procedures: A Systematic Review and Meta-analyses of Randomized Controlled Trials and Non-randomized Studies. World J Surg, 2017. 41(10): p. 2444-2456.

28. Raval, A.N., Cigarroa, J.E., Chung, M.K., Diaz-Sandoval, L.J., Diercks, D., Piccini, J.P., et al., Management of Patients on Non-Vitamin K Antagonist Oral Anticoagulants in the Acute Care and Periprocedural Setting: A Scientific Statement From the American Heart Association. Circulation, 2017. 135(10): p. e604-e633.

29. Pollack, C.V., Jr., Reilly, P.A., Eikelboom, J., Glund, S., Verhamme, P., Bernstein, R.A., et al., Idarucizumab for Dabigatran Reversal. N Engl J Med, 2015. 373(6): p. 511-20. 
30. Connolly, S.J., Milling, T.J., Jr., Eikelboom, J.W., Gibson, C.M., Curnutte, J.T., Gold, A., et al., Andexanet Alfa for Acute Major Bleeding Associated with Factor Xa Inhibitors. N Engl J Med, 2016. 375(12): p. 1131-41.

31. Madjdpour, C., Spahn, D.R., Weiskopf, R.B., Anemia and perioperative red blood cell transfusion: a matter of tolerance. Crit Care Med, 2006. 34(5 Suppl): p. S102-8.

32. Dai, J., Tu, W., Yang, Z., Lin, R., Case report: intraoperative management of extreme hemodilution in a patient with a severed axillary artery. Anesthesia and analgesia, 2010. 111(5): p. 1204-6.

33. Hebert, P.C., Carson, J.L., Transfusion threshold of $7 \mathrm{~g}$ per deciliter--the new normal. N Engl J Med, 2014. 371(15): p. 1459-61.

34. Spahn, D.R., Spahn, G.H., Stein, P., Evidence base for restrictive transfusion triggers in high-risk patients. Transfus Med Hemother, 2015. 42(2): p. 110-4.

35. Spahn, D.R., Patient Blood Management: the new standard. Transfusion, 2017. 57(6): p. 1325-1327. 